\title{
Pseudoconvexity on a closed convex set: an application to a wide class of generalized fractional functions
}

\section{Laura Carosi}

Received: date / Accepted: date

\begin{abstract}
The issue of the pseudoconvexity of a function on a closed set is addressed. It is proved that if a function has no critical points on the boundary of a convex set, then the pseudoconvexity on the interior guarantees the pseudoconvexity on the closure of the set. This results holds even when the boundary of the set contains line segments and it is used to characterize the pseudoconvexity, on the non-negative orthant, of a wide class of generalized fractional functions, namely the sum between a linear one and a ratio which has an affine function as numerator and, as denominator, the p-th power of an affine function. The relationship between quasiconvexity and pseudoconvexity is also investigated.
\end{abstract}

Keywords Pseudoconvexity · Quasiconvexity · Fractional Programming

Mathematics Subject Classification (2000) 90C32 26 B25

\section{Introduction}

Among different classes of generalized convex functions, pseudoconvexity plays a key role in Optimization theory and in many applied sciences such as Economics and Management Science. Pseudoconvexity owes its great relevance to the fact that it maintains some nice optimization properties of convex functions, such as critical and local minimum points are global minimum. Furthermore, if the objective function of a bicriteria problem is component-wise pseudoconvex, then the efficient frontier is connected.

Unlike their good properties, it is not easy to establish whether a function is pseudoconvex or not. Except for some particular classes of functions (see

Laura Carosi

Department of Economics and Management, University of Pisa, Italy

Tel.: +39-050-2216256

Fax: +39050 2210603

E-mail: laura.carosi@unipi.it 
for all the contributions of [2] and [7] together with the classical book [1] and references therein), pseudoconvexity characterizations are given for functions defined on an open and convex set; unfortunately these results can not be in general extended to the closure of the set itself. In this paper we will prove that if a function is pseudoconvex on the interior of a convex set and it has no critical points on the boundary, then it is pseudoconvex on the closure of the set. The stated result holds irrespective of the fact that the boundary contains line segments or not and it will be used to characterize the pseudoconvexity of a wide class of generalized fractional functions on the non-negative orthant. More precisely we will consider the sum between a linear function and a ratio which has an affine function as numerator and, as denominator, the $p$-th power $(p>0)$ of a positive affine one. Since the case $p=1$ has been recently analyzed $[5,9]$, we will focus our attention on $p>0, p \neq 1$. The performed analysis will be based on the second order characterization of pseudoconvexity given in [8] and it will give necessary and sufficient conditions for pseudoconvexity which are very easy to be verified. The stated conditions can also be used to construct several subclasses of pseudoconvex generalized fractional functions. Furthermore, we will analyze the relationship between the quasiconvexity and the pseudoconvexity of the proposed class of functions.

\section{Pseudoconvexity on a closed convex set}

For the sake of completeness we first recall the definition of pseudoconvexity and several useful properties (see for instance [3]).

Definition 1 Let $f$ be a differentiable function defined on an open set $A \subseteq \mathbb{R}^{n}$ and let $S \subseteq A$ be a convex set.

$f$ is said to be pseudoconvex on $S$ if the following implication holds:

$$
x^{1}, x^{2} \in S, \quad f\left(x^{1}\right)>f\left(x^{2}\right) \quad \Rightarrow \quad \nabla f\left(x^{1}\right)^{T}\left(x^{2}-x^{1}\right)<0
$$

Theorem 1 Let $f$ be a differentiable function defined on an open set $A \subseteq \mathbb{R}^{n}$ and let $S \subseteq A$ be a convex set.

i) A function $f$ is pseudoconvex on $S$ if and only if the restriction of $f$ on each line segment contained in $S$ is pseudoconvex.

ii) Let $\varphi$ be a restriction of $f$ on a line segment $I \subset S$.

$\varphi$ is pseudoconvex on $I$ if and only if for every $t_{0} \in I$ such that $\varphi^{\prime}\left(t_{0}\right)=0, t_{0}$ is a local minimum point of $\varphi$.

It is well known that the pseudoconvexity of a function on the interior of a closed convex set $X$ does not in general guarantee the pseudoconvexity on the whole set (see for instance [3]). We are going to prove that if there are no critical points on the boundary of $X$ (denoted by $\mathcal{F} r X$ ), the pseudoconvexity on the interior of $X$ implies the pseudoconvexity of the function on the whole set. 
Theorem 2 Let $f$ be a differentiable function on an open set $A \subseteq \mathbb{R}^{n}$ and let $X \subset A$ be a closed convex set, with int $X \neq \emptyset$.

If $f$ is pseudoconvex on $\operatorname{int} X$ and $\nabla f(x) \neq 0$ for every $x \in \mathcal{F} r X$, then $f$ is pseudoconvex on $X$.

Proof We will prove that $f$ is pseudoconvex on every line segment contained in $X$.

Let $x^{1}, x^{2} \in X$; with respect to the line segment $\left[x^{1}, x^{2}\right]$, we get either $\left(x^{1}, x^{2}\right) \subset$ $\operatorname{int} X$, or $\left[x^{1}, x^{2}\right] \subset \mathcal{F r} X$. Without any loss of generality, we assume, that $f\left(x^{1}\right)>f\left(x^{2}\right)$.

In the case $\left(x^{1}, x^{2}\right) \subset \operatorname{int} X$, we prove that $\nabla f\left(x^{1}\right)^{T}\left(x^{2}-x^{1}\right)<0$. If $x^{1}, x^{2} \in$ $\operatorname{int} X$, then the result follows from the definition of pseudoconvexity on int $X$; if otherwise, the continuity of $f$ implies the existence of a point $y \in\left(x^{1}, x^{2}\right)$ such that $f\left(x^{1}\right)>f(y)$. If $x^{1} \in \operatorname{int} X$, from the pseudoconvexity on $\operatorname{int} X$, it is $\nabla f\left(x^{1}\right)^{T}\left(y-x^{1}\right)<0$ and hence $\nabla f\left(x^{1}\right)^{T}\left(x^{2}-x^{1}\right)<0$. Suppose now that $x^{1} \in \mathcal{F} r X$; the assumptions imply $\nabla f\left(x^{1}\right) \neq 0$. If $\nabla f\left(x^{1}\right)^{T}\left(y-x^{1}\right)>0$, then $d=y-x^{1}$ is a local increasing direction; since $f\left(x^{1}\right)>f(y)$, the restriction $f\left(x^{1}+t d\right), t \in[0,1]$, has a maximum point belonging to the relative interior of the line segment $\left(x^{1}, y\right)$ and this contradicts the pseudoconvexity of the restriction of $f$ on $\left(x^{1}, y\right)$. If $\nabla f\left(x^{1}\right)^{T}\left(y-x^{1}\right)=0$, take $z=y+\epsilon \nabla f\left(x^{1}\right)$. For a suitable $\epsilon>0$, we have: $z \in \operatorname{int} X, f\left(x^{1}\right)>f(z)$, and $\nabla f\left(x^{1}\right)^{T}\left(z-x^{1}\right)=$ $\epsilon\left\|\nabla f\left(x^{1}\right)\right\|^{2}>0$. Once again, $f\left(x^{1}+t d^{1}\right), d^{1}=z-x^{1}, t \in[0,1]$, has a maximum point which belongs to int $X$ and this can not be true. Consequently $\nabla f\left(x^{1}\right)^{T}\left(y-x^{1}\right)<0$ and hence $\nabla f\left(x^{1}\right)^{T}\left(x^{2}-x^{1}\right)<0$.

Consider now the case $\left[x^{1}, x^{2}\right] \subset \mathcal{F} r X$ and let $\varphi(t)$ be the restriction of $f$ along the line segment $\left[x^{1}, x^{2}\right]$ with $\varphi(1)=x^{1}$ and $\varphi(0)=x^{2}$. Taking into account Theorem 1 , we are going to prove that if there exists $\bar{t} \in[0,1]$ such that $\varphi^{\prime}(\bar{t})=0$, then $\bar{t}$ is a minimum for $\varphi(t)$. If $x^{2}$ is the only critical point of $\varphi(t)$, that is $x^{2}=\bar{t}$, recalling that $\varphi(1)>\varphi(0), x^{2}$ is a minimum point.

Suppose now that there exists $\bar{t} \in(0,1]$ such that $\varphi^{\prime}(\bar{t})=0$. Let $\bar{x}=\bar{t} x^{1}+$ $(1-\bar{t}) x^{2}$ and take a point $y \in \operatorname{int} X$ such that $\nabla f(\bar{x})^{T}(y-\bar{x}) \neq 0$; consider the plane containing the points $\bar{x}, x^{2}, y$, that is $\Lambda=\left\{x \in \mathbb{R}^{n}: x=\right.$ $\left.\bar{x}+\alpha\left(\bar{x}-x^{2}\right)+\beta(y-\bar{x}), \alpha, \beta \in \mathbb{R}\right\}$. Obviously the line $r$ passing through $x^{1}, x^{2}$ is contained in $\Lambda$. Setting $\bar{\Lambda}=\Lambda \cap X$ and $\bar{r}=r \cap \bar{\Lambda}$, we get $\bar{r} \subset \mathcal{F} r \bar{\Lambda}$.

Let $\psi(\alpha, \beta)$ be the restriction of $f$ on $\bar{\Lambda}$; observe that there exists a suitable interval $I$ such that $\forall \alpha \in I \psi(\alpha, 0)$ coincides with $\varphi(t)$ and the point $\bar{t}$ corresponds to $\alpha=0$, so that $\frac{\partial \psi}{\partial \alpha}(0,0)=0$ and $\frac{\partial \psi}{\partial \beta}(0,0) \neq 0$. Since $\psi$ is pseudoconvex on the relative interior of $\bar{\Lambda}$, it is quasiconvex on $\bar{\Lambda}$ and hence the level set $\mathcal{L}=\{(\alpha, \beta): \psi(\alpha, \beta) \leq \psi(0,0)\}$ is convex (see for instance [3]).

Define $\mathcal{L}^{*}=\{(\alpha, \beta): \psi(\alpha, \beta)=\psi(0,0)\}$; if $\mathcal{L} \backslash \mathcal{L}^{*}=\emptyset$, then $(0,0)$ is a global minimum point for $\psi$ and, in particular, $\bar{t}$ is a minimum point for $\varphi$. If $\mathcal{L} \backslash \mathcal{L}^{*} \neq \emptyset,(0,0)$ is a boundary point for $\mathcal{L}$ and since $\frac{\partial \psi}{\partial \alpha}(0,0)=0$ and $\frac{\partial \psi}{\partial \beta}(0,0) \neq 0$, the line $s$ of equation $\beta=0$ is a support line for $\mathcal{L}$, so that $s \cap \operatorname{int} \mathcal{L}=\emptyset$. Consequently, for any point $(\alpha, 0)$, with $\alpha \in I$ it is 
$\psi(\alpha, 0) \geq \psi(0,0)$. It follows that $(0,0)$ is a minimum point for the restriction of $\psi$ along $\beta=0$, so that $\bar{t}$ is a minimum point for $\varphi$ and the proof is complete.

In the next section, Theorem 2 will be used to characterize the pseudoconvexity of a general class of fractional functions on the non-negative orthant of $\mathbb{R}^{n}$.

\section{Pseudoconvexity for a wide class of generalized fractional functions}

Consider the following class of generalized fractional functions

$$
f(x)=a^{T} x+\frac{c^{T} x+c_{0}}{\left(d^{T} x+d_{0}\right)^{p}}, \quad x \in D=\left\{x \in \mathbb{R}^{n}: d^{T} x+d_{0}>0\right\}
$$

Throughout the paper we will assume $d \in \operatorname{int} \mathbb{R}_{+}^{n}, d_{0}>0, p>0$ and $p \neq 1$ (For the case $p=1$ the interested reader can see $[5,9]$ ).

The gradient, the Hessian matrix $H(x)$ and the quadratic form associated with $H(x)$ are the following:

$$
\begin{gathered}
\nabla f(x)=a+\frac{c\left(d^{T} x+d_{0}\right)-p\left(c^{T} x+c_{0}\right) d}{\left(d^{T} x+d_{0}\right)^{p+1}} \\
H(x)=\frac{p}{\left(d^{T} x+d_{0}\right)^{p+2}}\left[\left(d^{T} x+d_{0}\right)\left(-d c^{T}-c d^{T}\right)+(p+1)\left(c^{T} x+c_{0}\right) d d^{T}\right] \\
w^{T} H(x) w=\frac{p}{\left(d^{T} x+d_{0}\right)^{p+2}}\left[-2\left(d^{T} x+d_{0}\right)\left(w^{T} d\right)\left(c^{T} w\right)+(p+1)\left(c^{T} x+c_{0}\right)\left(d^{T} w\right)^{2}\right]
\end{gathered}
$$

A key tool for our analysis will be the well-known second order characterization of pseudoconvexity on an open convex set, given in [8].

Theorem 3 Let $f$ be a twice continuously differentiable function defined on an open convex set $X \subseteq \mathbb{R}^{n}$.

Then, $f$ is pseudoconvex on $X$ if and only if the following conditions hold:

i) $x \in X, w \in \mathbb{R}^{n}, w^{T} \nabla f(x)=0 \Rightarrow w^{T} H(x) w \geq 0$;

ii) if $x_{0} \in X$ is a critical point, then $x_{0}$ is a local minimum point for $f$.

By means of the previous Theorem, we will prove that if the vectors $a, c, d$ are linearly independent, then function $f$ is never pseudoconvex.

Theorem 4 Let $S \subseteq D$ be a convex set with $\operatorname{int} S \neq \emptyset$. If $f$ is pseudoconvex on $S$, then $\operatorname{rank}[a, c, d] \leq 2$.

Proof Suppose on the contrary that $\operatorname{rank}[a, c, d]=3$; this implies $\nabla f(x) \neq 0$ for every $x \in S$. Applying Theorem 3, let us consider $x \in \operatorname{int} S$ and a direction $w$ such that $\nabla f(x)^{T} w=0$.

We have $a^{T} w+\frac{\left(d^{T} x+d_{0}\right) c^{T} w-p\left(c^{T} x+c_{0}\right) d^{T} w}{\left(d^{T} x+d_{0}\right)^{p+1}}=0$ so that

$$
\left(d^{T} x+d_{0}\right) c^{T} w=p\left(c^{T} x+c_{0}\right) d^{T} w-\left(d^{T} x+d_{0}\right)^{p+1} a^{T} w
$$


Substituting the value $c^{T} w\left(d^{T} x+d_{0}\right)$ in (5), we get

$$
w^{T} H(x) w=\frac{p}{\left(d^{T} x+d_{0}\right)^{p+2}} d^{T} w\left[(1-p)\left(c^{T} x+c_{0}\right) d^{T} w+2\left(d^{T} x+d_{0}\right)^{p+1} a^{T} w\right]
$$

For every $x \in \operatorname{int} S$, consider the linear map $A: \mathbb{R}^{n} \rightarrow \mathbb{R}^{3}, x \mapsto A x$, where $A=\left[\begin{array}{c}\nabla f(x)^{T} \\ a^{T} \\ d^{T}\end{array}\right]$. Since $\operatorname{rank}[a, c, d]=3$, the map A is surjective and hence we can choose $w \in \mathbb{R}^{n}$ such that $\nabla f(x)^{T} w=0, d^{T} w<0$ and $a^{T} w>\frac{(p-1)\left(c^{T} x+c_{0}\right)}{2\left(d^{T} x+d_{0}\right)^{p+1}} d^{T} w$, so that $w^{T} H(x) w<0$. Consequently, $f$ is not pseudoconvex on int $S$ and this is a contradiction.

\subsection{Pseudoconvexity on $\mathbb{R}_{+}^{n}$.}

Taking into account Theorem 4, the study of the pseudoconvexity of $f$ on the non-negative orthant $\mathbb{R}_{+}^{n}$ is carried on by considering the following exhaustive cases:

i) $\operatorname{rank}[a, c, d]=1$;

ii) $\operatorname{rank}[a, d]=2$, and $c, d$ are linearly dependent;

iii) $\operatorname{rank}[c, d]=2$, and $a$ is a linear combination of $c$ and $d$.

In the present paper we will not deal with the case $\operatorname{rank}[a, c, d]=1$ since, in this case, the study of the pseudoconvexity on $\mathbb{R}_{+}^{n}$ reduces to the study of the pseudoconvexity of a suitable one variable function and consequently it is very easy to handle (a complete discussion of the case $\operatorname{rank}[a, c, d]=1$ can be found in [6]). We are going to consider case ii) and iii) separately.

- Case $\operatorname{rank}[a, d]=2$, and $c, d$ are linearly dependent

The following theorem gives a complete characterization of the pseudoconvexity in Case ii).

Theorem 5 Assume $\operatorname{rank}[a, d]=2$ and $c=\beta d, \beta \in \mathbb{R}$.

Then $f$ is pseudoconvex on $\mathbb{R}_{+}^{n}$ if and only if the following conditions hold:

i) $\beta(p-1) \geq 0$;

ii) $\beta \leq \frac{c_{0}(p+1)}{2 d_{0}}$.

Proof The gradient and the Hessian matrix of $f$ become

$$
\begin{aligned}
& \nabla f(x)=a+\frac{d}{\left(d^{T} x+d_{0}\right)^{p+1}}\left[\beta(1-p)\left(d^{T} x+d_{0}\right)+p\left(\beta d_{0}-c_{0}\right)\right] \\
& H(x)=\frac{p d d^{T}}{\left(d^{T} x+d_{0}\right)^{p+2}}\left[\left(d^{T} x+d_{0}\right)(p-1) \beta+\left(c_{0}-\beta d_{0}\right)(p+1)\right]
\end{aligned}
$$


Note that $\nabla f(x) \neq 0, \forall x$; furthermore, $w^{T} H(x) w \geq 0$ for every $x \in \mathbb{R}_{+}^{n}$ if and only if $\left(d^{T} x+d_{0}\right)(p-1) \beta+\left(c_{0}-\beta d_{0}\right)(p+1) \geq 0, x \in \mathbb{R}_{+}^{n}$. It follows that necessarily we have $\beta(p-1) \geq 0$ and $\beta \leq \frac{c_{0}(p+1)}{2 d_{0}}$.

As a consequence of Theorem 5 we get the following equivalence.

Corollary 1 Assume $\operatorname{rank}[a, d]=2$ and $c=\beta d$.

Then $f$ is pseudoconvex on $\mathbb{R}_{+}^{n}$ if and only if $f$ is convex on $\mathbb{R}_{+}^{n}$.

- Case $\operatorname{rank}[c, d]=2$ and $a$ is a linear combination of $c$ and $d$

We will prove that, whenever $\nabla f(0) \neq 0$, the pseudoconvexity of $f$ on int $\mathbb{R}_{+}^{n}$ implies the pseudoconvexity of $f$ on $\mathbb{R}_{+}^{n}$ (Theorem 6 ). Exploiting the fact int $\mathbb{R}_{+}^{n}$ is open, we will able to apply Theorem 3 to obtain pseudoconvexity conditions (see Theorem 8 and Theorem 9). Finally we will see that when $\nabla f(0)=0, f$ may be pseudoconvex or not; nevertheless we will be able to characterize the pseudoconvexity of $f$ also in this particular case (see Theorem 10).

Referring to Theorem 3, two crucial aspects for our study are the existence (or non-existence) of critical points and the behavior of the Hessian matrix of $f$ along those directions which are orthogonal to the gradient. In this light, let us first compute the gradient of $f$, taking into account that $a$ is a linear combination of $c$ and $d$. Substituting $a=\alpha_{1} c+\alpha_{2} d$ in (3), we get

$$
\nabla f(x)=\frac{c\left(d^{T} x+d_{0}\right)\left(\alpha_{1}\left(d^{T} x+d_{0}\right)^{p}+1\right)+d\left(\alpha_{2}\left(d^{T} x+d_{0}\right)^{p+1}-p\left(c^{T} x+c_{0}\right)\right)}{\left(d^{T} x+d_{0}\right)^{p+1}}
$$

Since $\operatorname{rank}[c, d]=2, f$ has critical points in $\mathbb{R}_{+}^{n}$ if and only if the following system has solutions in $\mathbb{R}_{+}^{n}$ :

$$
\begin{cases}\alpha_{1}\left(d^{T} x+d_{0}\right)^{p}+1 & =0 \\ \alpha_{2}\left(d^{T} x+d_{0}\right)^{p+1}-p\left(c^{T} x+c_{0}\right) & =0\end{cases}
$$

Consider the following function

$$
h(x)=(1-p) \frac{\left(c^{T} x+c_{0}\right)}{\left(d^{T} x+d_{0}\right)^{p}}+\left(\alpha_{1}(p+1) c+2 \alpha_{2} d\right)^{T} x+\alpha_{1}(p+1) c_{0}+2 \alpha_{2} d_{0}
$$

The following lemma plays a fundamental role both in proving the non-existence of critical points on $\mathbb{R}_{+}^{n} \backslash\{0\}$ and in characterizing the pseudoconvexity of $f$ on int $\mathbb{R}_{+}^{n}$.

Lemma 1 Assume $\operatorname{rank}[c, d]=2$.

i) $f$ is pseudoconvex on $D_{1}^{+}=\left\{x \in \operatorname{int} \mathbb{R}_{+}^{n}: \alpha_{1}\left(d^{T} x+d_{0}\right)^{p}+1>0\right\}$ if and only if $h(x) \geq 0, \forall x \in D_{1}^{+}$.

ii) $f$ is pseudoconvex on $D_{1}^{-}=\left\{x \in \operatorname{int} \mathbb{R}_{+}^{n}: \alpha_{1}\left(d^{T} x+d_{0}\right)^{p}+1<0\right\}$ if and only if $h(x) \leq 0, \forall x \in D_{1}^{-}$. 
Proof Note that $\alpha_{1}\left(d^{T} x+d_{0}\right)^{p}+1 \neq 0$ implies the non-existence of critical points, so that we can refer to i) of Theorem 3. Condition $w^{T} \nabla f(x)=0$ holds if and only if

$\left(d^{T} x+d_{0}\right) c^{T} w=\frac{1}{\left(\alpha_{1}\left(d^{T} x+d_{0}\right)^{p}+1\right)} d^{T} w\left(p\left(c^{T} x+c_{0}\right)-\alpha_{2}\left(d^{T} x+d_{0}\right)^{p+1}\right)$

Substituting (10) in (5) we get

$$
w^{T} H(x) w=\frac{p\left(d^{T} w\right)^{2}}{\left(d^{T} x+d_{0}\right)^{2}} \frac{h(x)}{\alpha_{1}\left(d^{T} x+d_{0}\right)^{p}+1}
$$

Therefore $w^{T} H(x) w \geq 0$ if and only if $\frac{h(x)}{\alpha_{1}\left(d^{T} x+d_{0}\right)^{p}+1} \geq 0$. The proof is complete.

By means of the previous Lemma and taking into account Theorem 2 we get the following fundamental result.

Theorem 6 Assume $\operatorname{rank}[c, d]=2$.

i) If $f$ is pseudoconvex on int $\mathbb{R}_{+}^{n}$, then $\nabla f(x) \neq 0$ for every $x \in \mathbb{R}_{+}^{n} \backslash\{0\}$.

ii) If $\nabla f(0) \neq 0$, then $f$ is pseudoconvex on $\mathbb{R}_{+}^{n}$ if and only if $f$ is pseudoconvex on $\operatorname{int} \mathbb{R}_{+}^{n}$.

iii) If $f$ is pseudoconvex on $\mathbb{R}_{+}^{n}$, then either $\alpha_{1} \geq 0$ or $\alpha_{1} \leq-\frac{1}{d_{0}^{p}}$.

Proof We will prove that $\alpha_{1}\left(d^{T} x+d_{0}\right)^{p}+1 \neq 0$ for every $x \in \mathbb{R}_{+}^{n} \backslash\{0\}$. By contradiction assume the existence of $\bar{x} \in \mathbb{R}_{+}^{n} \backslash\{0\}$ such that $\alpha_{1}\left(d^{T} \bar{x}+d_{0}\right)^{p}+$ $1=0$. Since $d \in \operatorname{int} \mathbb{R}_{+}^{n}$ and $d_{0}>0$, both $D_{1}^{+}$and $D_{1}^{-}$are non empty. Taking into account Lemma 1 and the continuity of $h$, it results $h(x)=0$ for every $x \in \operatorname{int} \mathbb{R}_{+}^{n}$ such that $\alpha_{1}\left(d^{T} x+d_{0}\right)^{p}+1=0$. Substituting $\alpha_{1}=-\frac{1}{\left(d^{T} x+d_{0}\right)^{p}}$ in $h(x)=0$ we get $\alpha_{2}\left(d^{T} x+d_{0}\right)^{p+1}-p\left(c^{T} x+c_{0}\right)=0$, so that, from (8), $f$ has critical points belonging to int $\mathbb{R}_{+}^{n}$. Due to the linear independence of $c$ and $d$, the quadratic form (5) is indefinite for every fixed $x \in \mathbb{R}_{+}^{n}$ and hence any critical point of $f$ in $\operatorname{int} \mathbb{R}_{+}^{n}$ is not a minimum point and this contradicts the pseudoconvexity of $f$.

Conditions $\alpha_{1}\left(d^{T} x+d_{0}\right)^{p}+1 \neq 0$ for every $x \in \mathbb{R}_{+}^{n} \backslash\{0\}$ implies that $f$ admits no critical points in $\mathbb{R}_{+}^{n} \backslash\{0\}$ and that either $\alpha_{1} \geq 0$ or $\alpha_{1} \leq-\frac{1}{d_{0}^{p}}$, so that i) and iii) hold.

ii) follows directly from i) and Theorem 2 .

From Theorem 6 and Lemma 1, the study of the pseudoconvexity of $f$ reduces to give conditions on the sign of $h$; more precisely if $\alpha_{1} \geq 0\left(\alpha_{1} \leq-\frac{1}{d_{0}^{p}}\right)$ we will give conditions guaranteeing $h$ is non-negative ( $h$ is non-positive). 
Note that, by continuity, condition $h(x) \geq 0(h(x) \leq 0), \forall x \in \operatorname{int} \mathbb{R}_{+}^{n}$, implies $h(x) \geq 0(h(x) \leq 0), \forall x \in \mathbb{R}_{+}^{n}$, or, equivalently, $\inf _{x \in \mathbb{R}_{+}^{n}} h(x) \geq 0\left(\sup _{x \in \mathbb{R}_{+}^{n}} h(x) \leq 0\right)$. The following theorem states that the sign of $h$ on $\mathbb{R}_{+}^{n}$ can be deduced by the sign of the restriction of $h$ on the edges of $\mathbb{R}_{+}^{n}$.

Theorem 7 There exists an index $i \in I=\{1, \ldots, n\}$ such that

$$
\begin{aligned}
& \inf _{x \in \mathbb{R}_{+}^{n}} h(x)=\inf _{x_{i} \geq 0} h_{i}\left(x_{i}\right) \\
& \sup _{x \in \mathbb{R}_{+}^{n}} h(x)=\sup _{x_{i} \geq 0} h_{i}\left(x_{i}\right)
\end{aligned}
$$

where $h_{i}\left(x_{i}\right)$ denotes the restriction of function $h(x)$ on the $i$-th edge of $\mathbb{R}_{+}^{n}$.

Proof Let $\left\{x_{n}\right\} \subset \mathbb{R}_{+}^{n}$ be a sequence such that $h\left(x_{n}\right) \rightarrow \ell=\inf _{x \in \mathbb{R}_{+}^{n}} h(x)$.

For every fixed $x_{n}$, consider the linear problem

$$
P_{n}: \inf _{x \in S_{n}} h(x), \quad S_{n}=\left\{x \in \mathbb{R}_{+}^{n}: d^{T} x+d_{0}=d^{T} x_{n}+d_{0}\right\}
$$

Since $S_{n}$ is a compact set, the infimum is attained as a minimum at a vertex $\hat{x}_{n}$, which belongs to an edge of $\mathbb{R}_{+}^{n}$, and it is $h\left(\hat{x}_{n}\right) \leq h\left(x_{n}\right), \forall n$. Consequently, $h\left(\hat{x}_{n}\right) \rightarrow \ell$. The finite number of edges implies the existence of a subsequence $\left\{y_{n}\right\}$ of $\left\{x_{n}\right\}$, contained in an edge, such that $h\left(y_{n}\right) \rightarrow \ell$, so that (12) holds. The proof of (13) follows in a similar way.

Remark 1 When $a=0$, that is $\alpha_{1}=\alpha_{2}=0, h(x)$ reduces to $h(x)=$ $\frac{(1-p)\left(c^{T} x+c_{0}\right)}{\left(d^{T} x+d_{0}\right)^{p}}$, so that $f$ is pseudoconvex on $\operatorname{int} \mathbb{R}_{+}^{n}$ if and only if $0<p<1$, $c \in \mathbb{R}_{+}^{n}, c_{0} \geq 0$ or $p>1, c \in \mathbb{R}_{-}^{n}, c_{0} \leq 0$.

From now on, taking into account the previous remark, we will consider the case $a \neq 0$. Furthermore, for sake of simplicity, we will assume $c \in \operatorname{int} \mathbb{R}_{+}^{n}$. Conditions for the case $c_{i} \leq 0$ can be obtained following the same strategies used in the results that we are going to present.

In order to characterize the pseudoconvexity of $f$ on int $\mathbb{R}_{+}^{n}$, according with iii) of Theorem 6 , we first deal with the case $\alpha_{1} \geq 0$.

Theorem 8 Assume $\operatorname{rank}[c, d]=2, c \in \operatorname{int} \mathbb{R}_{+}^{n}$ and $\alpha_{1} \geq 0$.

Then $f$ is pseudoconvex on int $\mathbb{R}_{+}^{n}$ if and only if:

i) $\alpha_{1}(p+1) c+2 \alpha_{2} d \in \mathbb{R}_{+}^{n} \backslash\{0\}$;

ii) $(1-p) c_{0}+d_{0}^{p}\left(\alpha_{1}(p+1) c_{0}+2 \alpha_{2} d_{0}\right) \geq 0$

and one of the following conditions holds:

iii) $0<p<1$;

iv) $p>1$ and either $\nabla h(0)=(1-p) \frac{d_{0} c-p c_{0} d}{d_{0}^{p+1}}+\alpha_{1}(p+1) c+2 \alpha_{2} d \in \mathbb{R}_{+}^{n}$, or

$\min _{i \in J} h_{i}\left(\bar{x}_{i}\right) \geq 0$, where $J=\left\{i: \frac{\partial h}{\partial x_{i}}(0)<0\right\}$, and $\bar{x}_{i}$ is such that $h_{i}^{\prime}\left(\bar{x}_{i}\right)=0$. 
Proof The assumption $\alpha_{1} \geq 0$ guarantees the non-existence of critical points, so that $f$ is pseudoconvex on int $\mathbb{R}_{+}^{n}$ if and only if $h(x) \geq 0, \forall x \in \operatorname{int} \mathbb{R}_{+}^{n}$.

Firstly we prove that $i$ ) and $i i$ ) are necessary conditions for pseudoconvexity. Note that $h(x) \geq 0, \forall x \in \operatorname{int} \mathbb{R}_{+}^{n}$, implies $h(0) \geq 0$, i.e., $\left.i i\right)$. On the other hand, if $\alpha_{1}(p+1) c+2 \alpha_{2} d \notin \mathbb{R}_{+}^{n} \backslash\{0\}$, then there exists a restriction of $h$ on an edge of $\mathbb{R}_{+}^{n}$ for which $\inf _{x_{i} \geq 0} h_{i}\left(x_{i}\right)=-\infty$ and this is a contradiction.

Let us now consider the case $0<p<1$. Conditions i) and ii) imply that $h_{i}\left(x_{i}\right)$, $i=1, \ldots, n$, is the sum of increasing functions with $h_{i}(0) \geq 0$. From Theorem 7 we get $h(x) \geq 0$, for every $x \in \operatorname{int} \mathbb{R}_{+}^{n}$.

We are left to prove that conditions i), ii) and iv) imply $h(x) \geq 0, \forall x \in \operatorname{int} \mathbb{R}_{+}^{n}$, or, equivalently (see Theorem 7 ), $h_{i}\left(x_{i}\right) \geq 0, \forall x_{i} \geq 0, \forall i \in\{1, \ldots, n\}$.

We have

$$
h_{i}^{\prime}\left(x_{i}\right)=(1-p) \frac{(1-p) c_{i} d_{i} x_{i}+c_{i} d_{0}-p d_{i} c_{0}}{\left(d_{i} x_{i}+d_{0}\right)^{p+1}}+\alpha_{1}(p+1) c_{i}+2 \alpha_{2} d_{i}
$$

and

$$
h_{i}^{\prime \prime}\left(x_{i}\right)=\frac{(1-p) p}{\left(d_{i} x_{i}+d_{0}\right)^{p+2}} d_{i}\left[(p-1) c_{i} d_{i} x_{i}-2 c_{i} d_{0}+(p+1) d_{i} c_{0}\right]
$$

Let us preliminary observe that $h_{i}^{\prime}\left(x_{i}\right)$ has a unique critical point which is a maximum point and $\lim _{x_{i} \rightarrow+\infty} h_{i}^{\prime}\left(x_{i}\right)=\alpha_{1}(p+1) c_{i}+2 \alpha_{2} d_{i} \geq 0$. Therefore if $\nabla h(0) \in \mathbb{R}_{+}^{n}$, that is $h_{i}^{\prime}(0) \geq 0$ for every $i$, then $h_{i}^{\prime}\left(x_{i}\right) \geq 0, \forall x_{i} \geq 0$. Since $h_{i}(0) \geq 0$, it results $h_{i}\left(x_{i}\right) \geq 0, \forall x_{i} \geq 0$.

On the other hand, if $i \in J$, then there exists a critical point $\bar{x}_{i}$ which is a minimum for $h_{i}$ with $h_{i}\left(\bar{x}_{i}\right) \geq 0$. Consequently $h_{i}\left(x_{i}\right) \geq 0, \forall x_{i} \geq 0, \forall i \in J$ and the thesis follows.

Remark 2 When $0<p<1$, condition i) of Theorem 8 implies $a_{i} \geq \frac{(1-p) c_{i}}{2}$. Consequently, if there exists $i$ such that $a_{i}<0$, then $f$ is not pseudoconvex.

The following example points out that, in the case $J \neq \emptyset$, condition $\min _{i \in J} h_{i}\left(\bar{x}_{i}\right) \geq$ 0 can not be relaxed.

Example 1 Consider the function $f(x, y)=\frac{1}{32} x+\frac{4 x+y-1}{(3 x+y+1)^{2}}$. The restriction of $f$ on $y=1$ has a critical point at $x=0,98142397$ which is a maximum point. Therefore $f$ is not pseudoconvex on int $\mathbb{R}_{+}^{2}$.

Referring to Theorem 8 , it is easy to verify that $a=\frac{1}{32} c-\frac{1}{32} d$ and $\operatorname{rank}[c, d]=$ 2. Since $\alpha_{1}=\frac{1}{32}>0, f$ has no critical points. Moreover, necessary conditions i) and ii) of Theorem 8 are verified.

On the other hand, $h_{1}(x)=h(x, 0)=-\frac{(4 x-1)}{(3 x+1)^{2}}+\frac{3}{16} x-\frac{5}{32}$ with $h_{1}^{\prime}(0)=$ $-\frac{157}{16}$, hence $J \neq \emptyset$. Function $h_{1}(x)$ has a minimum point at $\bar{x}=0,546247734$ with $h_{1}(\bar{x})=-0,224$, so that condition iv) of Theorem 8 is not verified. 
From iii) of Theorem 6 , to completely characterize the pseudoconvexity of $f$ on $\operatorname{int} \mathbb{R}_{+}^{n}$ it remains to deal with the case $\alpha_{1} \leq-\frac{1}{d_{0}^{p}}$.

Theorem 9 Assume $\operatorname{rank}[c, d]=2, c \in \operatorname{int} \mathbb{R}_{+}^{n}$ and $\alpha_{1} \leq-\frac{1}{d_{0}^{p}}$.

Then $f$ is pseudoconvex on int $\mathbb{R}_{+}^{n}$ if and only if:

i) $\alpha_{1}(p+1) c+2 \alpha_{2} d \in \mathbb{R}_{-}^{n} \backslash\{0\}$;

ii) $(1-p) c_{0}+d_{0}^{p}\left(\alpha_{1}(p+1) c_{0}+2 \alpha_{2} d_{0}\right) \leq 0$;

and one of the following conditions holds:

iii) $p>1$;

iv) $0<p<1$ and $\max _{i \notin J_{1}} h_{i}\left(\bar{x}_{i}\right) \leq 0$, where $J_{1}=\left\{i: \frac{\partial h}{\partial x_{i}}(0) \leq 0, \frac{c_{i}}{d_{i}} \geq \frac{p+1}{2} \frac{c_{0}}{d_{0}}\right\}$ and $\bar{x}_{i}$ is such that $h_{i}^{\prime}\left(\bar{x}_{i}\right)=0$

Proof Note that the assumption $\alpha_{1} \leq-\frac{1}{d_{0}^{p}}$ guarantees the non-existence of critical points, consequently $f$ is pseudoconvex on int $\mathbb{R}_{+}^{n}$ if and only if $h(x) \leq 0, \forall x \in \mathbb{R}_{+}^{n}$.

Firstly we prove that $i$ ) and $i i)$ are necessary conditions for pseudoconvexity. Infact, $h(x) \leq 0, \forall x \in \mathbb{R}_{+}^{n}$, implies $h(0) \leq 0$, i.e., condition $\left.i i\right)$. On the other hand, if $\alpha_{1}(p+1) c+2 \alpha_{2} d \notin \mathbb{R}_{-}^{n} \backslash\{0\}$, there exists a restriction on an edge of $\mathbb{R}_{+}^{n}$ for which $h(x) \rightarrow+\infty$ and this is a contradiction.

Let us now consider the case $p>1$. Condition i) and ii) imply that $h_{i}\left(x_{i}\right)$, $i=1, \ldots, n$, is the sum of decreasing functions with $h_{i}(0) \leq 0$. From Theorem 7 we get $h(x) \leq 0$, for every $x \in \operatorname{int} \mathbb{R}_{+}^{n}$.

We are left to prove that when $0<p<1$, conditions i), ii) and iv) imply $h(x) \leq 0, \forall x \in \operatorname{int} \mathbb{R}_{+}^{n}$, or, equivalently (see Theorem 7 ), $h_{i}\left(x_{i}\right) \leq 0, \forall x_{i} \geq 0$, $\forall i \in\{1, \ldots, n\}$.

Let us note that $\lim _{x_{i} \rightarrow+\infty} h_{i}^{\prime}\left(x_{i}\right)=\alpha_{1}(p+1) c_{i}+2 \alpha_{2} d_{i} \leq 0$.

If $i \in J_{1}$, then from (15) we get $h_{i}^{\prime}\left(x_{i}\right) \leq 0, \forall x_{i} \geq 0$; consequently, $h_{i}\left(x_{i}\right) \leq$ $0, \forall x_{i} \geq 0$.

Consider now the case $i \notin J_{1}$.

If $h_{i}^{\prime}(0)>0$, then $h_{i}$ has a maximum point $\bar{x}_{i}$, so that condition $\max _{i \notin J_{1}} h_{i}\left(\bar{x}_{i}\right) \leq 0$ implies $h_{i}\left(x_{i}\right) \leq 0, \forall x_{i} \geq 0$.

If $h_{i}^{\prime}(0)<0$, and $\frac{c_{i}}{d_{i}}<\frac{p+1}{2} \frac{c_{0}}{d_{0}}, h_{i}^{\prime}$ has a maximum point at $\tilde{x}=\frac{2 c_{i} d_{0}-(p+1) d_{i} c_{0}}{c_{i} d_{i}(p-1)}$.

If $h_{i}^{\prime}(\tilde{x}) \leq 0$, then $h_{i}$ is decreasing so that $h_{i}\left(x_{i}\right) \leq 0, \forall x_{i} \geq 0$. If $h_{i}^{\prime}(\tilde{x})>0$, then there exists $\bar{x}_{i}$ such that $h_{i}^{\prime}\left(\bar{x}_{i}\right)=0$ which is a maximum point for $h_{i}$. Condition $\max _{i \notin J_{1}} h_{i}\left(\bar{x}_{i}\right) \leq 0$ implies $h_{i}\left(x_{i}\right) \leq 0, \forall x_{i} \geq 0$. The proof is complete.

Remark 3 When $0<p<1$, condition i) of Theorem 9 implies $a_{i} \leq \frac{(1-p) c_{i}}{2}$. Consequently, if there exists $i$ such that $a_{i}>0$, then $f$ is not pseudoconvex.

The following example points out that condition $\max _{i \notin J_{1}} h_{i}\left(\bar{x}_{i}\right) \leq 0$ can not be relaxed. 
Example 2 Consider the function $f(x, y)=-1,001 x-3,001 y+\frac{2 x+3 y+8}{\sqrt{x+y+1}}$, the point $P=(898,1)$ and the direction $w^{T}=\left(w_{1}, w_{2}\right)$ with $w_{1}=-1212,843229$ and $w_{2}=400$. It can be verified that $\nabla f(P)^{T} w=0$, and $w^{T} H(P) w<0$. Therefore $f$ is not pseudoconvex on int $\mathbb{R}_{+}^{2}$.

Referring to Theorem 9 , it is $a=-2 c+2,999 d$ and $\operatorname{rank}[c, d]=2$. Since $\alpha_{1}=-2<-1, f$ has no critical points and, by simple computations, it follows that necessary conditions i) and ii) of Theorem 9 are verified. Moreover $h_{1}^{\prime}(0)<0$ and $\frac{c_{1}}{d_{1}}=2<\frac{p+1}{2} \frac{c_{0}}{d_{0}}=6$, so that $1 \notin J_{1}$. On the other hand, the maximum value of $h_{1}$ is positive and so $f$ is not pseudoconvex.

Summarizing the previous results, whenever $\nabla f(0) \neq 0$ the pseudoconvexity on $\mathbb{R}_{+}^{n}$ is completely characterized by the pseudoconvexity on $\operatorname{int} \mathbb{R}_{+}^{n}$ (see i) and ii) of Theorem 6); moreover Theorem 8 and Theorem 9 provide a complete characterization of the pseudoconvexity of $f$ on $\operatorname{int} \mathbb{R}_{+}^{n}$ and hence, in the case $\nabla f(0) \neq 0$, on $\mathbb{R}_{+}^{n}$. Therefore we are left to deal with the case $\nabla f(0)=0$.

The particular case $\nabla f(0)=0$

The following example shows that, when the origin is a critical point, $f$ may be not pseudoconvex.

Example 3 Consider the function $f(x, y)=-\frac{1}{125} x-\frac{9}{500} y+\frac{x+2 y+1}{(x+y+10)^{2}}$.

It is to verify that $\alpha_{1}=-\frac{1}{100}$ and $\alpha_{2}=\frac{1}{500}$ and conditions i), ii) and iii) of Theorem 9 hold, so that $f$ is pseudoconvex on $\mathbb{R}_{+}^{2} \backslash\{0\}$. Moreover $(0,0)$ is a critical point and it is a maximum for the restriction of $f$ on the half-line $(0, y), y \geq 0$. Therefore $f$ is not pseudoconvex on $\mathbb{R}_{+}^{2}$.

The following theorem provides necessary and sufficient conditions for the pseudoconvexity of $f$ when the origin is a critical point. Note that $\nabla f(0)=0$ if and only if $\alpha_{1}=-\frac{1}{d_{0}^{p}}$ and $\alpha_{2}=\frac{p c_{0}}{d_{0}^{p+1}}$.

Theorem 10 Assume $\operatorname{rank}[c, d]=2, c \in \operatorname{int} \mathbb{R}_{+}^{n}, \alpha_{1}=-\frac{1}{d_{0}^{p}}$ and $\alpha_{2}=\frac{p c_{0}}{d_{0}^{p+1}}$.

Then, $f$ is pseudoconvex on $\mathbb{R}_{+}^{n}$ if and only if the following conditions hold:

i) $p>1$;

ii) $\frac{2 p}{p+1} \frac{c_{0}}{d_{0}} \leq \min _{i} \frac{c_{i}}{d_{i}}$

iii) $\max _{i} \frac{c_{i}}{d_{i}} \leq \frac{p+1}{2} \frac{c_{0}}{d_{0}}$.

Proof Assume that $f$ is pseudoconvex. Condition ii) follows immediately from i) of Theorem 9 . 
Consider now the restriction $\varphi_{u}(t)$ of $f$ on the half-line $x=t u, t \geq 0, u \in \mathbb{R}_{+}^{n}$. We have

$$
\begin{array}{r}
\varphi_{u}^{\prime}(t)=\frac{p c_{0} d^{T} u-d_{0} c^{T} u}{d_{0}^{p+1}}+\frac{(1-p) c^{T} u d^{T} u t+d_{0} c^{T} u-p c_{0} d^{T} u}{\left(t d^{T} u+d_{0}\right)^{p+1}} \\
\varphi_{u}^{\prime \prime}(t)=\frac{p d^{T} u}{\left(t d^{T} u+d_{0}\right)^{p+2}}\left[(p-1) c^{T} u d^{T} u t-2 d_{0} c^{T} u+(p+1) c_{0} d^{T} u\right]
\end{array}
$$

Since $\varphi_{u}^{\prime}(0)=0$, necessarily we must have $\varphi_{u}^{\prime \prime}(0) \geq 0$, i.e. $\frac{c^{T} u}{d^{T} u} \leq \frac{p+1}{2} \frac{c_{0}}{d_{0}}, \forall u \in$ $\mathbb{R}_{+}^{n}$ which is equivalent to iii). We are left to show that $p>1$.

Suppose that $0<p<1$; since ii) holds, there exists $u \in \mathbb{R}_{+}^{n}$ such that $\frac{c^{T} u}{d^{T} u}>\frac{p c_{0}}{d_{0}}$ and hence $\lim _{t \rightarrow+\infty} \varphi_{u}^{\prime}(t)<0$. Moreover, from (16) and from condition iii), $\varphi_{u}^{\prime}(t)$ has a maximum point $\tilde{t}>0$. Since $\varphi_{u}^{\prime}(\tilde{t})>0, \varphi_{u}^{\prime}$ has one zero corresponding to a maximum point for $\varphi_{u}(t)$, contradicting the pseudoconvexity of $f$.

Viceversa, assume that conditions i), ii) and iii) hold.

From Theorem $9, f$ is pseudoconvex on $\operatorname{int} \mathbb{R}_{+}^{n}$ and hence from Theorem $6, f$ is pseudoconvex on $\mathbb{R}_{+}^{n} \backslash\{0\}$. It remains to prove that the restriction of $f$ on every half-line of the kind $x=t u, t \geq 0, u \in \mathbb{R}_{+}^{n}$ is pseudoconvex; observe that conditions $p>1$ and $\frac{c^{T} u}{d^{T} u} \leq \frac{p+1}{2} \frac{c_{0}}{d_{0}}, \forall u \in \mathbb{R}_{+}^{n}$, guarantee the convexity of every restriction $\varphi_{u}(t)$ and this completes the proof.

\section{On the relationship between pseudoconvexity and quasiconvexity}

We would like to make some remarks on the relationship between pseudoconvexity and quasiconvexity. As it is well known the following properties hold (see for instance [3]):

1. if a function $f$ is pseudoconvex, then $f$ is quasiconvex;

2. if $\nabla f(x) \neq 0$, for every $x$ belonging to an open convex set $S$, then $f$ is pseudoconvex if and only if it is quasiconvex.

It is worth pointing out that Theorem 2 extends Property 2. to a function which is defined on a closed convex set.

Corollary 2 Let $f$ be a differentiable function on an open set $A \subseteq \mathbb{R}^{n}$ and let $X \subset A$ be a closed convex set, with $\operatorname{int} X \neq \emptyset$.

If $\nabla f(x) \neq 0$ for every $x \in X$, then $f$ is pseudoconvex on $X$ if and only if $f$ is quasiconvex.

With respect to the considered class of generalized fractional functions, the following theorem provides a sufficient condition which guarantees the equivalence between the pseudoconvexity and the quasiconvexity on an open convex set. 
Theorem 11 Consider the function $f(x)=a^{T} x+\frac{c^{T} x+c_{0}}{\left(d^{T} x+d_{0}\right)^{p}}$ and let $S$ be an open and convex set contained in $D$.

If $\operatorname{rank}[a, c, d]=2$, then $f$ is pseudoconvex on $S$ if and only if $f$ is quasiconvex on $S$.

Proof If $\operatorname{rank}[a, d]=2$ and $c=\beta d$, it is $\nabla f(x) \neq 0$ for every $x \in S$ (see (6)), so that the thesis follows from Property 2 . If $\operatorname{rank}[c, d]=2$ we prove that the quasiconvexity of $f$ implies the non-existence of critical points. Suppose by contradiction that $f$ admits a critical point $x^{0}$; since $\operatorname{rank}[c, d]=2, H\left(x^{0}\right)$ is indefinite. Let $u$ be an eigenvector associated with a negative eigenvalue of $H\left(x^{0}\right)$ and let $\varphi$ be the restriction of $f$ on the line segment $x=x^{0}+t u$, $t \in(-\epsilon, \epsilon) ; \varphi$ admits a maximum point and this is a contradiction. Therefore $f$ has not critical points and the thesis follows again from Property 2 .

The previous result does not in general hold if we consider an arbitrary closed convex set $S$. Actually the following examples point out that whenever $f$ admits critical points, the proper inclusion still holds.

Example 4 Consider the function $f(x)=3 x+\frac{36 x+3}{(4 x+1)^{2}}$ on the set $X=[0,+\infty)$. $f$ is quasiconvex on $X$ since it is an increasing function; on the other hand, it admits a critical point at $x=\frac{1}{4}$ which is not a minimum point, so that $f$ is not pseudoconvex.

Example 5 Referring to Example $3, f$ is not pseudoconvex on $\mathbb{R}_{+}^{2}$, but it is quasiconvex, since $f$ is pseudoconvex on int $\mathbb{R}_{+}^{2}$.

\section{Concluding remarks}

In this paper the equivalence between the pseudoconvexity of a function on the interior of a convex set and the pseudoconvexity on the closure of the set is established. This result is applied to characterize the pseudoconvexity on $\mathbb{R}_{+}^{n}$ of a wide class of generalized fractional functions. The obtained conditions are very easy to be verified and suggest further developments. From an algorithmic point of view, in [4], the authors propose a procedure for solving problems having this kind of functions as objective and a polyhedron as feasible region. The new pseudoconvexity characterizations, obtained in the present paper, can be applied to improve the procedure in [4]; whenever the function is pseudoconvex, new stop criteria can be considered. As soon as a local minimum is reached, the global minimum point is found and the procedure can stop without further investigations. Furthermore, the particular structure of the problem and the new pseudoconvexity characterizations would suggest to conceive also simplex-like sequential methods for solving this class of problems. Moving from the scalar to the bicriteria case, it would be interesting to derive the efficient frontier when one of the two objectives is linear and the other one belongs to the studied class. 
Acknowledgements The author would like to thank the anonymous referees for their valuable remarks and suggestions. Moreover, the author would like to express her sincere appreciation and gratitude to Professors Laura Martein and Alberto Cambini for their comments and fruitful support.

\section{References}

1. Avriel, M., Diewert, W.E., Schaible, S., Zang, I.: Generalized concavity, vol. 63. Siam (2010). (originally published by Plenum Press, New York, 1988)

2. Avriel, M., Schaible, S.: Second order characterizations of pseudoconvex functions. Mathematical Programming 14(1), 170-185 (1978)

3. Cambini, A., Martein, L.: Generalized Convexity and Optimization: Theory and Applications, Lecture Notes in Economics and Mathematical Systems, vol. 616. Springer, Berlin Heidelberg (2009)

4. Cambini, R., Sodini, C.: A unifying approach to solve some classes of rank-three multiplicative and fractional programs involving linear functions. European Journal of Operational Research 207(1), 25-29 (2010)

5. Carosi, L., Martein, L.: A sequential method for a class of pseudoconcave fractional problems. Central European Journal of Operations Research 16(2), 153-164 (2008)

6. Carosi, L., Martein, L.: Characterizing the pseudoconvexity of a wide class of generalized fractional functions. Tech. rep., University of Pisa, Department of Economics and Management, Italy (2013)

7. Cottle, R.W., Ferland, J.A.: Matrix-theoretic criteria for the quasi-convexity and pseudoconvexity of quadratic functions. Linear Algebra and its Applications 5(2), 123-136 (1972)

8. Crouzeix, J.P., Ferland, J.A.: Criteria for quasi-convexity and pseudo-convexity: relationships and comparisons. Mathematical Programming 23(1), 193-205 (1982)

9. Martein, L., Carosi, L.: The sum of a linear and a linear fractional function: pseudoconvexity on the nonnegative orthant and solution methods. Bulletin of the Malaysian Mathematical Sciences Society 35(2A) (2012) 